\title{
Necrotizing panniculitis secondary to primary cutaneous localized mucormycosis
}

\begin{abstract}
Primary cutaneous mucormycosis is a rare entity that affects more frequently extremities of immunosuppressed patients and may be gradual in onset or fulminant.

An 83-year-old female with diabetes mellitus presented a nodular lesion in the forearm, which had progressed during one month. Clinically, as the lesion was indolent and followed a linear trajectory, a superficial phlebitis was suspected but later ruled out. Skin blood cultures were negative. Skin biopsy showed a necrotic panniculitis with isolated ghost adipocytes and presence of irregularly branched hyphae involving dermis and some vessels that could be stained with PAS and Grocott, thus leading to the diagnosis of primary cutaneous localized mucormycosis. This case is noteworthy as indolent course in primary cutaneous mucormycosis with vascular involvement is rather rare. Even with a negative culture, $R$. Variabilis is a candidateetiologic agent as it usually presents a chronic evolution Histopathologically, most panniculitis related to fungal infection are reportedas pancreatic panniculitis mimickers. In our case, only scarce ghost adipocytes were found and the main histopathological findings were a lobular panniculitis with vasculitis and an extensive basophilic necrosis. Thus, these histopathologic findings should raise suspicion of infection related-panniculitis, and PAS and Grocott stainings should be performed to rule out fungi.
\end{abstract}

Volume 5 Issue 2 - 202 I

\author{
P Chicharro,' E Gallo,' E Daudén,' J Fraga,² \\ M Llamas Velasco' \\ 'Department of Dermatology, Hospital Universitario de la \\ Princesa, Spain \\ ${ }^{2}$ Department of Pathology, Hospital Universitario de la Princesa, \\ Spain
}

Correspondence: M Llamas Velasco, Department of Dermatology, Hospital Universitario de la Princesa, Spain Tel +34915202433, Email mar.llamasvelasco@gmail.com

Received: March 23, 202I | Published: April 20, 2021

Keywords: mucormycosis, diabetes mellitus, diabetes complications, panniculitis, thrombophlebitis

\section{Learning Points}

Primary cutaneous mucormycosis is a rare entity and in some cases the clinical lesions can be quite indolent. R. variabilis is usually involved in indolent lesions.

Cultures may be negative despite the presence of a large number of fungal structures.

Skin biopsy can be the leading diagnostic test showing necrotic panniculitis with ghost adipocytes and hyphae surrounding and invading vascular structures that can be detected with PAS and Grocott staining.

\section{Introduction}

Mucormycosis is a rare fungal infection secondary to opportunistic fungi of the phylum Glomeromycota, subphylum Mucormycotina, which are ubiquitous in nature. Clinical presentations include rhinocerebral, cutaneous, pulmonary, disseminated and gastrointestinal as well as miscellaneous cases. ${ }^{1}$ Cutaneous mucormicosis portends $8.41 \%$ of mucormycosis cases in a 35 -year retrospective study in a tertiary-care center. ${ }^{2}$ Cutaneous mucormycosis can be primary, or secondary when involvement depends ondissemination from other locations. As an example ilncidence in Spain of cutaneous mucormycosis was 0.43 cases per millionin habitants per year in 2005. Although it is more frequent in immunocompromised hosts such as diabetic patients, some cases may occur in immunocompetent patients, because of a damage in the skin, secondary to direct inoculation, contaminated dressing, surgery or burns, which leads the fungal structures to invade dermis and even hypodermis. ${ }^{1}$

Our objective is to show an indolent case of primary cutaneous mucormycosis to discuss several challenges on how to perform the diagnosis.

\section{Case report}

Our patient wasan 83-year-old female with type 2 diabetes mellitus and diabetic nephropathy whowas in the intensive unit care(Hospital Universitario de la Princesa, Madrid, Spain) due to a hemorrhagic shock after a coronary surgery. Her intensive care treatment included corticosteroids, prophylactic antibiotic and antifungal drugs. She referred the presence,since one month before the surgery, of a slightly itchy nodular lesion, with progressive enlargement. Clinical examination showed a nodular lesion with an overlying linear violaceous area in the right forearm (Figure 1). Despite the clinical history, as the lesion seemed to follow a venous trajectory, we performed an Eco-Doppler to rule out superficial phlebitis. Eco-Doppler only showed the presence of hyperechogenic subcutaneous tissue with an increase of vascularization, thus suggesting panniculitis. Skin biopsy showed a necrotic panniculitis with isolated ghost adipocytes and presence of irregularly branched hyphae between them involving vascular structures (Figure 2 a-c). PAS and Grocott staining showed thick non-septated hyphae (Figure 2 d). Skin and blood cultures were negative. As the patient passed away due to her hemorrhagic shock, we couldnot perform additional skin or blood cultures.

The final diagnosis was necrotizing panniculitis secondary to mucor infection clinically mimicking superficial thrombophlebitis.

\section{Discussion}

Primary cutaneous mucormycosis is a rare entity. Clinically, lesionsare mostly deep necrotic ulcers, affect more frequently arms and legs and may be gradual in onset or fulminant.

Our case is worthy as lesions showed a very indolent course even when fungi invaded the vessels. This situation completely differs from self-resolving superficial mucormycosis reported in very superficial fungal infections. ${ }^{4}$ Although the patient and her family did 
not recall the cause, direct inoculation would be the more plausible wayin a predisposed patient. Cultures were negative, a rare finding, but primary cutaneous mucormycosis by $\mathrm{R}$. variabilis, unlike other mucorales, presents as chronic infections with infiltrated plaques, ulcers and nodules, which usually remain localized and gradually expand over months and years. ${ }^{1,5}$ In our case, the absence of growth in culture may be due to agitation or use of media different from Sabouraud or potato dextrose agar. ${ }^{6,7}$

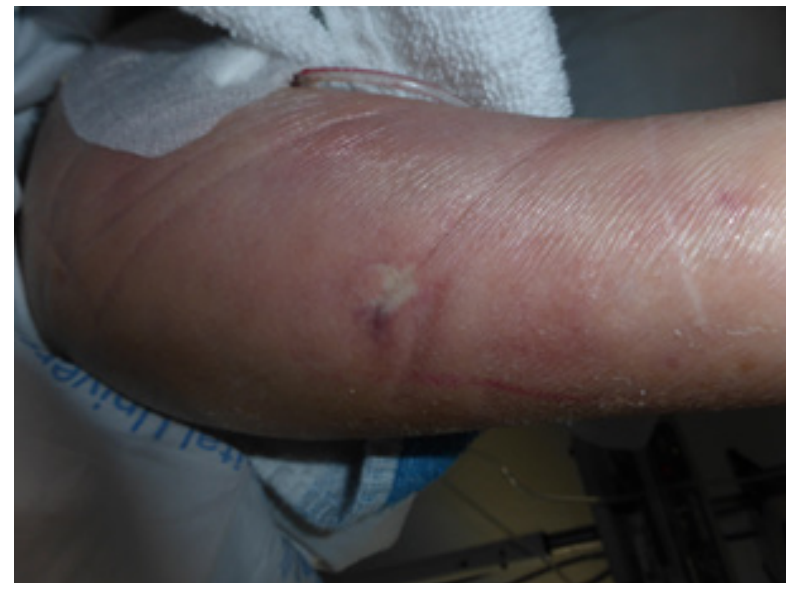

Figure I Erythematous and edematous redness in the forearm with presence of a centrally located nodule.A slightly indurated linear plaque can be palpated proximal to the nodule.

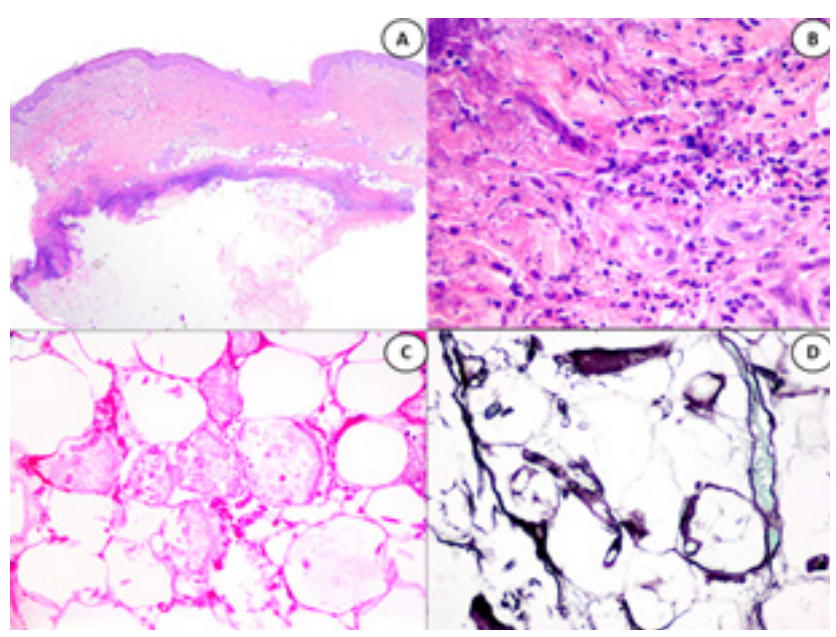

Figure 2 (A) Panoramic picture of HE staining showing massive adipocyte necrosis as well as a horizontally arranged violaceous area in the dermohypodermal area (B) HExI0. Detail of the violaceous area, showing basophilic necrosis as well as neutrophil infiltration $(C) \mathrm{HExIO}$ showing ghost adipocytes with some intermingled fungal structures. (D) Grocott staining showing non-septated hyphae.

Regarding skin biopsy, histopathologic features of mucoraceae infection in panniculitisare characterized by necrosis as well as areas of ghost adipocytes mimicking the ones observed in pancreatic panniculitis, ${ }^{8}$ which can be similar in cases related to aspergillosis. ${ }^{9}$ Grocott also highlights the thick, hyaline, non-septated and bifurcated hyphae in cutaneous mucormycosis. These histopathological findings are caused by the fungal production of extracellular lipases, the synthesis of oxalic acid and the increase of uric acid due to necrotic tissues. Even without these features, a lobular panniculitis with vascular damage and basophilic necrosis, as in our patient, may raise suspicion of infective panniculitis and should be studied with cultures or special stains. ${ }^{10}$ Regarding prognosis, localized mucormycosis mortality ranged from 4 to $10 \%$, much lower than the overall mortality of all mucormycosis that is $47 \%$ in 1990 after the wide use of amphotericin B, that is the elective drug. Surgical removal of the infected tissue can be added when possible. ${ }^{11}$ In cases with renal failure, as our patient, posaconazol and isavuconazole may be used but it is important to emphasize that itraconazole has limited activity and voriconazole is not active $e^{1,12}$ We could not start specific treatment as the patientpassed away due to the hemorrhagic shock.

To summarize, primary localized mucormycosis is a rare entity that requires a high level of suspicion to be diagnosed. This diagnosis should be taken into account in cases with any predisposition factor, even with slowly progressing lesions. Although microbiologic isolation of the fungi remains the gold standard, histopathologic studies are very useful to get a fast and specific diagnosis. We present an atypical clinical presentation where skin biopsy showing a necrotic panniculitis where fungal structures could be observed with HE, PAS and Grocott staining, led to the final diagnosis. Our case contributes toexpand the wide variety of clinical morphologies of primary cutaneous mucormycosis.

\section{Conflicts of interest}

There are no conflicts of interest.

\section{Acknowledgments}

None.

Funding

None.

\section{References}

1. Castrejon-Perez AD, Welsh EC, Miranda I, et al. Cutaneous mucormycosis. An Bras Dermatol. 2017;92;304-311.

2. Bonifaz A, Tirado-Sanchez A, Hernandez-Medel ML, et al Mucormycosis at a tertiary-care center in mexico. A 35-year retrospective study of 214 cases. Mycoses. 2021;64(4);372-380.

3. Bonifaz A, Tirado-Sanchez A, Hernandez-Medel ML, et al. Mucormycosis with cutaneous involvement. A retrospective study of 115 cases at a tertiary care hospital in mexico. Australas J Dermatol. 2020.

4. Jones MU, Flores MS, Vereen RJ, et al. Self-resolving superficial primary cutaneous mucormycosis in a 7-week-old infant. Pediatr Dermatol. 2018;35(4);e248-e250.

5. Xia XJ, Shen H, Liu ZH. Primary cutaneous mucormycosis caused by mucor irregularis. Clin Exp Dermatol. 2015;40(8);875-878.

6. Walsh TJ, Gamaletsou MN, McGinnis MR, et al. Early clinical and laboratory diagnosis of invasive pulmonary, extrapulmonary, and disseminated mucormycosis (zygomycosis). Clin Infect Dis. 2012;54 Suppl 1;S55-60.

7. Skiada A, Rigopoulos D, Larios G, et al. Global epidemiology of cutaneous zygomycosis. Clin Dermatol. 2012;30(6);628-632.

8. Requena L, Sitthinamsuwan $\mathrm{P}$, Santonja $\mathrm{C}$, et al. Cutaneous and mucosal mucormycosis mimicking pancreatic panniculitis and gouty panniculitis. J Am Acad Dermatol. 2012;66(6);975-984. 
9. Colmenero I, Alonso-Sanz M, Casco F, et al. Cutaneous aspergillosis mimicking pancreatic and gouty panniculitis. J Am Acad Dermatol. 2012;67(4);789-791.

10. Chan MP. Neutrophilic panniculitis: Algorithmic approach to a heterogeneous group of disorders. Arch Pathol Lab Med. 2014;138(10):1337-1343.

11. Priya P, Ganesan V, Rajendran T, et al. Mucormycosis in a tertiary care center in south india: A 4-year experience. Indian J Crit Care Med. 2020;24(3);168-171.
12. Blyth CC, Gilroy NM, Guy SD, et al. Consensus guidelines for the treatment of invasive mould infections in haematological malignancy and haemopoietic stem cell transplantation, 2014. Intern Med J. $2014 ; 44(12 b) ; 1333-1349$ 